\title{
EXISTENCE OF POSITIVE SOLUTIONS OF PBVPS FOR FIRST-ORDER DIFFERENCE EQUATIONS
}

\author{
YINGGAO ZHOU
}

Received 5 January 2006; Accepted 13 March 2006

We consider the existence of positive solutions for the following first-order periodic boundary value problem: $x(n+1)=x(n)-f(n, x(n)), 0 \leq n \leq \omega-1, x(0)=x(\omega)$. Some criteria for existence of positive solutions of the above difference boundary problem are established by using Krasnosel'skiîs's fixed point theorem, and some multiplicity results of positive solutions are also derived.

Copyright (c) 2006 Yinggao Zhou. This is an open access article distributed under the Creative Commons Attribution License, which permits unrestricted use, distribution, and reproduction in any medium, provided the original work is properly cited.

\section{Introduction}

Consider the following periodic boundary value problem (PBVP for short):

$$
\begin{gathered}
x(n+1)=x(n)-f(n, x(n)), \quad 0 \leq n \leq \omega-1, \\
x(0)=x(\omega),
\end{gathered}
$$

where $\omega$ is a positive integer, $f(n, x)$ is defined on $\mathbb{Z} \times \mathbb{R}$ and is $\omega$-periodic with regard to $n$.

In recent years, the existence of periodic solutions of boundary value problems for difference equations has been studied extensively, see, for example, [1, 2, 4, 6-8]. In [5], Peng investigated the existence of positive solutions for the related differential boundary problem of PBVP (1.1) by applying a fixed point theorem in a cone. To the knowledge of the authors, there are very few works on the existence of positive solutions for PBVP (1.1). In this paper, we establish a group of conditions to guarantee PBVP (1.1) to have positive solutions by applying the Krasnosel'skir fixed point theorem in a cone.

Throughout this paper, we denote the product of $y(n)$ from $n=a$ to $n=b$ by $\prod_{n=a}^{b} y(n)$ with the understanding that $\prod_{n=a}^{b} y(n)=1$ for all $a>b$, and we give some restricted conditions. 
2 Existence of positive solutions of PBVPs

(H1) There exists an $\omega$-periodic sequence $a(n)$ such that $0<a(n)<1, a(n) x \geq f(n, x)$, for all $x \geq 0,0 \leq n \leq \omega-1$.

(H2) For any $\varepsilon>0$, there exists $\delta>0$ such that $\|x-y\|<\delta$ implies that $\mid f(n, x)-$ $f(n, y) \mid<\varepsilon$, for all $0 \leq n \leq \omega-1$.

\section{Preliminaries}

Consider the following PBVP for first-order linear difference equation:

$$
\begin{gathered}
x(n+1)=r(n) x(n)+g(n), \quad 0 \leq n \leq \omega-1, \\
x(0)=x(\omega),
\end{gathered}
$$

where $r: \mathbb{Z} \rightarrow \mathbb{R}^{+}$with $0<r(n)<1,0 \leq n \leq \omega-1, g$ is a sequence.

Let

$$
G(n, s)= \begin{cases}\frac{\prod_{t=s+1}^{n-1} r(t)}{1-\prod_{t=0}^{\omega-1} r(t)}, & 0 \leq s \leq n-1, \\ \frac{\prod_{t=0}^{n-1} r(t) \prod_{t=s+1}^{\omega-1} r(t)}{1-\prod_{t=0}^{\omega-1} r(t)}, & n \leq s \leq \omega-1 .\end{cases}
$$

Note that the denominator in $G(n, s)$ is not zero since $0<r(n)<1$ for $0 \leq n \leq \omega-1$. We can easily claim that the following lemma holds.

Lemma 2.1. For any sequence $g, P B V P(2.1)$ has a unique solution

$$
x(n)=\sum_{s=0}^{\omega-1} G(n, s) g(s)
$$

Clearly, let $g(n)=1-r(n)$, it holds that $\sum_{s=0}^{\omega-1} G(n, s)(1-r(s))=1$. Let

$$
C_{\omega}=\{x(n): \mathbb{Z} \longrightarrow \mathbb{R}, x(n+\omega)=x(n)\} .
$$

Then it is clear that $C_{\omega}$ is a Banach space endowed the norm $\|x\|=\sup _{0 \leq n \leq \omega-1}|x(n)|$.

For any $u \in C_{\omega}$, consider the following PBVP:

$$
\begin{gathered}
x(n+1)=(1-a(n)) x(n)+[a(n) u(n)-f(n, u(n))], \quad 0 \leq n \leq \omega-1, \\
x(0)=x(\omega) .
\end{gathered}
$$

From Lemma 2.1, we will know that PBVP (2.5) has a unique solution

$$
x_{u}(n)=\sum_{s=0}^{\omega-1} G(n, s) g_{u}(s)=\sum_{s=0}^{\omega-1} G(n, s)[a(s) u(s)-f(s, u(s))],
$$

where $G(n, s)$ is defined as in (2.2), $r(n)=1-a(n), g_{u}(n)=a(n) u(n)-f(n, u(n))$. Let $\sigma=\prod_{t=0}^{\omega-1} r(t)=\prod_{t=0}^{\omega-1}(1-a(t))$. Now, define a cone by

$$
P=\left\{x(n) \in C_{\omega}: x(n) \geq \sigma\|x\|\right\},
$$


meanwhile, we define an operator $\Phi: C_{\omega} \rightarrow C_{\omega}$ as

$$
(\Phi u)(n)=\sum_{s=0}^{\omega-1} G(n, s) g_{u}(s)
$$

By (2.5), it is easy to verify that $u=u(n) \in C_{\omega}$ is a solution of (1.1) provided $u$ is a fixed point of $\Phi$.

Lemma 2.2. The mapping $\Phi$ maps $P$ into $P$, that is, $\Phi P \subset P$.

Proof. It is easy to see that

$$
A:=\frac{\sigma}{1-\sigma} \leq G(n, s) \leq \frac{1}{1-\sigma}:=B
$$

From (2.8) and (2.9), we have, for $x \in P$,

$$
\begin{gathered}
\|(\Phi u)\| \leq B \sum_{s=0}^{\omega-1} g_{u}(s), \\
(\Phi u)(n) \geq A \sum_{s=0}^{\omega-1} g_{u}(s) \geq \frac{A}{B}\|(\Phi u)\| \geq \sigma\|(\Phi u)\| .
\end{gathered}
$$

Hence, $\Phi P \subset P$. The proof is complete.

Lemma 2.3. $\Phi: P \rightarrow P$ is completely continuous.

Proof. By (H2), for $x, y \in C_{\omega}$, and $\|x-y\|<\delta$,

$$
\begin{aligned}
\|\Phi x-\Phi y\| & \leq \sum_{s=0}^{\omega-1}|G(n, s)|(\|a\| \cdot\|x-y\|+(1-\|a\|)\|x-y\|) \\
& \leq B \omega\|x-y\| .
\end{aligned}
$$

Hence, $\Phi$ is continuous. On the other hand, $\Phi$ is a bounded and finite-dimensional operator because of periodicity of $x, r$ and $f$. Therefore, the operator $\Phi$ is completely continuous. The proof is complete.

\section{Existence of positive solutions}

To conclude this section, we introduce the definition of cone and the well-known Krasnosel'skiu fixed point theorem which will be needed in this paper.

Definition 3.1. Let $X$ be a Banach space and let $P$ be a closed, nonempty subset of $X . P$ is a cone if

(i) $\alpha x+\beta y \in P$ for all $x, y \in P$ and all $\alpha, \beta \geq 0$;

(ii) $x,-x \in P$ imply $x=0$. 


\section{Existence of positive solutions of PBVPs}

Lemma 3.2 (Krasnosel'skiu [3]). Let $X$ be a Banach space, and let $P \subset X$ be a cone in $X$. Assume that $\Omega_{1}, \Omega_{2}$ are open bounded subsets of $X$ with $0 \in \Omega_{1} \subset \bar{\Omega}_{1} \subset \Omega_{2}$, and let

$$
\varphi: P \cap\left(\bar{\Omega}_{2} \backslash \Omega_{1}\right) \longrightarrow P
$$

be a completely continuous operator such that either

(i) $\|\varphi x\| \leq\|x\|$, for all $x \in P \cap \partial \Omega_{1}$ and $\|\varphi x\| \geq\|x\|$, for all $x \in P \cap \partial \Omega_{2}$, or

(ii) $\|\varphi x\| \geq\|x\|$, for all $x \in P \cap \partial \Omega_{1}$ and $\|\varphi x\| \leq\|x\|$, for all $x \in P \cap \partial \Omega_{2}$.

Then $\varphi$ has a fixed point in $P \cap\left(\bar{\Omega}_{2} \backslash \Omega_{1}\right)$.

Theorem 3.3. Assume that (H1) and (H2) hold, and that there exist two positive number $M, N$ with $M \neq \sigma^{-1} N$ such that

$$
\begin{gathered}
f(n, x) \leq 0, \quad \sigma^{-1} N \geq x \geq N \\
f(n, x) \geq 0, \quad \sigma M \leq x \leq M .
\end{gathered}
$$

Then PBVP (1.1) has at least one positive solution.

Proof. Suggest that, without loss of generality, $M<\sigma^{-1} N$, we define

$$
\Omega_{1}=\left\{x(n) \in C_{\omega}:\|x\|<M\right\} .
$$

If $u(n) \in P \cap \partial \Omega_{1}$, then $\sigma\|u\| \leq u(n) \leq\|u\|=M$, and

$$
\|\Phi u\| \leq \sum_{s=0}^{\omega-1} G(n, s) a(s) M=\|u\| .
$$

Next, we define

$$
\Omega_{2}=\left\{x(n) \in C_{\omega}:\|x\|<\sigma^{-1} N\right\} .
$$

If $u(n) \in P \cap \partial \Omega_{2}$, then $N=\sigma\|u\| \leq u(n) \leq\|u\|=\sigma^{-1} N$, and

$$
\|\Phi u\| \geq \sum_{s=0}^{\omega-1} G(n, s) a(s)\|u\|=\|u\| .
$$

By Lemma 3.2, $\Phi$ has a positive fixed point $u(n)$ with $u(n) \geq \sigma M>0$, and $u(n)$ is a positive solution of PBVP (1.1). This completes the proof.

Corollary 3.4. Assume that (H1) and (H2) hold, and that there exist two positive numbers $M \ll N$ such that

$$
\max _{0 \leq n \leq \omega-1} f(n, x) \leq 0, \quad x \geq N, \quad \min _{0 \leq n \leq \omega-1} f(n, x) \geq 0, \quad x \leq M,
$$

or

$$
\max _{0 \leq n \leq \omega-1} f(n, x) \leq 0, \quad x \leq N, \quad \min _{0 \leq n \leq \omega-1} f(n, x) \geq 0, \quad x \geq M .
$$

Then PBVP (1.1) has at least one positive solution. 
Corollary 3.5. Assume that (H1) and (H2) hold, and that

$$
\limsup _{x \rightarrow+\infty} f(n, x)<0, \quad \liminf _{x \rightarrow 0^{+}} f(n, x)>0,
$$

or

$$
\limsup _{x \rightarrow 0^{+}} f(n, x)<0, \quad \liminf _{x \rightarrow+\infty} f(n, x)>0 .
$$

Then PBVP (1.1) has at least one positive solution.

Similar to the proof of Theorem 3.3, we also have the following theorem (Theorem 3.6).

TheOREm 3.6. Assume that (H1) and (H2) hold, and that there exist two positive numbers $d, h$ with $d \neq h$ such that

$$
\begin{array}{ll}
f(n, x) \leq a(n)(x-h), & \sigma h \geq x \geq h, \\
f(n, x) \geq a(n)(x-d), & \sigma d \leq x \leq d .
\end{array}
$$

Then PBVP (1.1) has at least one positive solution.

Theorem 3.7. Assume that (H1) and (H2) hold, and that the following condition holds:

(H3) for $0 \leq a \leq x \leq b, 0 \leq n \leq \omega-1, f(n, b) \leq f(n, x) \leq f(n, a)$.

If the inequalities

$$
\begin{gathered}
\min _{0 \leq n \leq \omega-1} \sum_{s=0}^{\omega-1} G(n, s)[a(s) x-f(s, x)] \geq \frac{1}{\sigma} x, \\
\max _{0 \leq n \leq \omega-1} \sum_{s=0}^{\omega-1} G(n, s)[a(s) x-f(s, x)] \leq x
\end{gathered}
$$

have positive solutions $u^{*}$ and $v^{*}$, respectively, and that $u^{*} \neq \sigma v^{*}$, then (1.1) has at least one positive solution.

Proof. For $u^{*}>\sigma v^{*}$, we define

$$
\Omega_{1}=\left\{x(n) \in C_{\omega}:\|x\|<v^{*}\right\} .
$$

If $x(n) \in P \cap \partial \Omega_{1}$, then $\sigma\|x\| \leq x(n) \leq\|x\|=v^{*}$, and

$$
\begin{aligned}
\|\Phi x\| & \leq \max _{0 \leq n \leq \omega-1} \sum_{s=0}^{\omega-1} G(n, s)[a(s) x(s)-f(s, x(s))] \\
& \leq \max _{0 \leq n \leq \omega-1} \sum_{s=0}^{\omega-1} G(n, s)\left[a(s) v^{*}-f\left(s, v^{*}\right)\right] \\
& \leq v^{*}=\|x\| .
\end{aligned}
$$

Next, we define

$$
\Omega_{2}=\left\{x(n) \in C_{\omega}:\|x\|<\sigma^{-1} u^{*}\right\} .
$$


6 Existence of positive solutions of PBVPs

If $x(n) \in P \cap \partial \Omega_{2}$, then $\sigma\|x\| \leq x(n) \leq\|x\|=\sigma^{-1} u^{*}$, and

$$
\begin{aligned}
\|\Phi x\| & \geq \min _{0 \leq n \leq \omega-1} \sum_{s=0}^{\omega-1} G(n, s)[a(s) x(s)-f(s, x(s))] \\
& \geq \min _{0 \leq n \leq \omega-1} \sum_{s=0}^{\omega-1} G(n, s)\left[a(s) u^{*}-f\left(s, u^{*}\right)\right] \\
& \geq \sigma^{-1} u^{*}=\|x\| .
\end{aligned}
$$

Obviously, $\Omega_{1}$ and $\Omega_{2}$ are open bounded subsets of $C_{\omega}$ with $0 \in \Omega_{1} \subset \bar{\Omega}_{1} \subset \Omega_{2}$. Hence, $\Phi: P \cap\left(\bar{\Omega}_{2} \backslash \Omega_{1}\right) \rightarrow P$ is a completely continuous operator and satisfies condition (i) in Lemma 3.2. By Lemma 3.2, there exists a point $x^{*}=x^{*}(t) \in P \cap\left(\bar{\Omega}_{2} \backslash \Omega_{1}\right)$ such that $x^{*}(t)=$ $\left(\Phi x^{*}\right)(t) \cdot x^{*}(t)$ is a positive solution of (2.8). For $u^{*}<\sigma v^{*}$, the proof can be similarly obtained by invoking condition (ii) of Lemma 3.2. This completes the proof.

Corollary 3.8. Assume that (H1)-(H3) hold, and that the inequalities

$$
\begin{gathered}
\sum_{s=0}^{\omega-1}[a(s) x-f(s, x)] \geq \sigma^{-1}\left(\sigma^{-1}-1\right) x, \\
\sum_{s=0}^{\omega-1}[a(s) x-f(s, x)] \leq(1-\sigma) x
\end{gathered}
$$

have positive solutions $u^{*}$ and $v^{*}$, respectively, and that $u^{*} \neq \sigma v^{*}$. Then (1.1) has at least one positive solution.

Proof. We have

$$
\begin{aligned}
A \sum_{s=0}^{\omega-1}[a(s) x-f(s, x)] & \leq \min _{0 \leq n \leq \omega-1} \sum_{s=0}^{\omega-1} G(n, s)[a(s) x-f(s, x)] \\
& \leq \max _{0 \leq n \leq \omega-1} \sum_{s=0}^{\omega-1} G(n, x)[a(s) x-f(s, x)] \leq B \sum_{s=0}^{\omega-1}[a(s) x-f(s, x)],
\end{aligned}
$$

which implies that (3.12) hold. This complete the proof.

\section{Multiplicity of positive solutions}

Theorem 4.1. Assume that (H1) and (H2) hold. If

$$
\limsup _{x \rightarrow 0^{+}} f(n, x)<0, \quad \limsup _{x \rightarrow+\infty} f(n, x)<0,
$$

and there exists a $p>0$ such that $\sigma p<x<p$ implies that

$$
f(n, x) \geq 0,
$$

then PBVP (1.1) has at least two positive solutions. 
Proof. By (4.1), there exist $R_{1}>0, R_{2}>0\left(R_{1}\right.$ is small enough, and $R_{2}$ is large enough, $R_{1}<p<R_{2}$ ), such that

$$
\begin{gathered}
f(n, x) \leq 0, \quad 0 \leq x \leq R_{1}, \\
f(n, x) \leq 0, \quad x \geq R_{2} .
\end{gathered}
$$

From Theorem 3.3, (4.2) and (4.4) imply that PBVP (1.1) has at least one positive solution, so are (4.2) and (4.3). The proof is complete.

Similar to the proof of Theorem 4.1, we have the following theorem.

Theorem 4.2. Assume that (H1) and (H2) hold. If

$$
\liminf _{x \rightarrow 0^{+}} f(n, x)>0, \quad \liminf _{x \rightarrow+\infty} f(n, x)>0,
$$

and there exists a $p>0$ such that $\sigma p<x<p$ implies that

$$
f(n, x) \leq 0
$$

then PBVP (1.1) has at least two positive solutions.

Corollary 4.3. Assume that (H1) and (H2) hold. If

$$
\limsup _{x \rightarrow 0^{+}} \frac{f(n, x)}{x}<0, \quad \limsup _{x \rightarrow+\infty} \frac{f(n, x)}{x}<0,
$$

and there exists a $p>0$ such that $\sigma p<x<p$ implies that

$$
f(n, x) \geq 0,
$$

then PBVP (1.1) has at least two positive solutions.

Corollary 4.4. Assume that (H1) and (H2) hold. If

$$
\liminf _{x \rightarrow 0^{+}} \frac{f(n, x)}{x}>0, \quad \liminf _{x \rightarrow+\infty} \frac{f(n, x)}{x}>0,
$$

and there exists a $p>0$ such that $\sigma p<x<p$ implies that

$$
f(n, x) \leq 0
$$

then PBVP (1.1) has at least two positive solutions.

\section{Acknowledgment}

This work is partially supported by the NNSF(10471153) of China. 


\section{Existence of positive solutions of PBVPs}

\section{References}

[1] R. P. Agarwal and F.-H. Wong, Existence of positive solutions for higher order difference equations, Applied Mathematics Letters 10 (1997), no. 5, 67-74.

[2] D. R. Anderson, Discrete third-order three-point right-focal boundary value problems, Computers \& Mathematics with Applications 45 (2003), no. 6-9, 861-871.

[3] M. A. Krasnosel'skiı̌, Positive Solutions of Operator Equations, P. Noordhoff, Groningen, 1964.

[4] W.-T. Li and J. P. Sun, Existence of positive solutions of BVPs for third-order discrete nonlinear difference systems, Applied Mathematics and Computation 157 (2004), no. 1, 53-64.

[5] S. Peng, Positive solutions for first order periodic boundary value problem, Applied Mathematics and Computation 158 (2004), no. 2, 345-351.

[6] J.-P. Sun and W.-T. Li, Existence of positive solutions of boundary value problem for a discrete difference system, Applied Mathematics and Computation 156 (2004), no. 3, 857-870.

[7] P. J. Y. Wong and R. P. Agarwal, Double positive solutions of $(n, p)$ boundary value problems for higher order difference equations, Computers \& Mathematics with Applications 32 (1996), no. 8, $1-21$.

[8] B. G. Zhang, L. J. Kong, Y. J. Sun, and X. H. Deng, Existence of positive solutions for BVPs of fourth-order difference equations, Applied Mathematics and Computation 131 (2002), no. 2-3, $583-591$.

Yinggao Zhou: School of Mathematical Sciences and Computing Technology,

Central South University, Changsha, Hunan 410083, China

E-mail address: ygzhou@mail.csu.edu.cn 


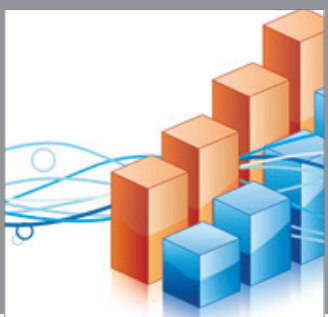

Advances in

Operations Research

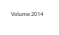

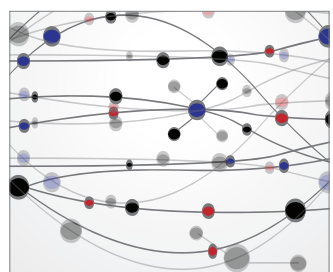

\section{The Scientific} World Journal
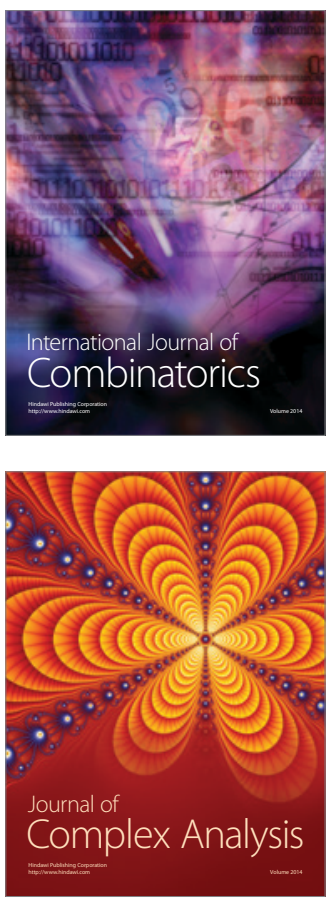

International Journal of

Mathematics and

Mathematical

Sciences
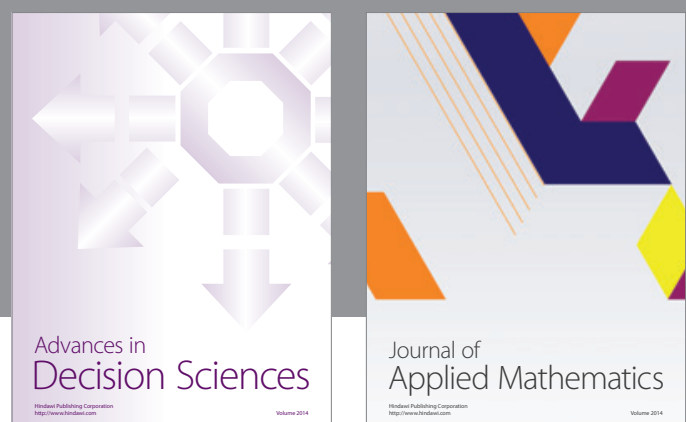

Journal of

Applied Mathematics
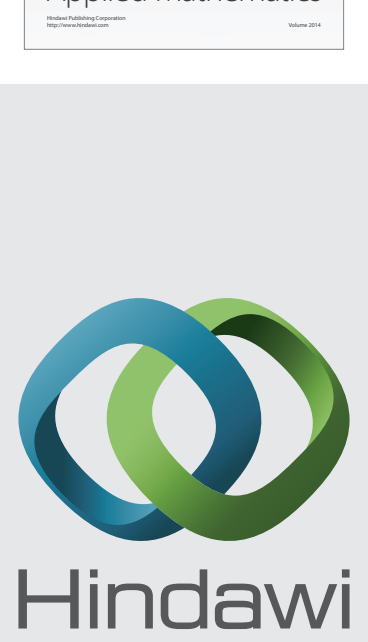

Submit your manuscripts at http://www.hindawi.com
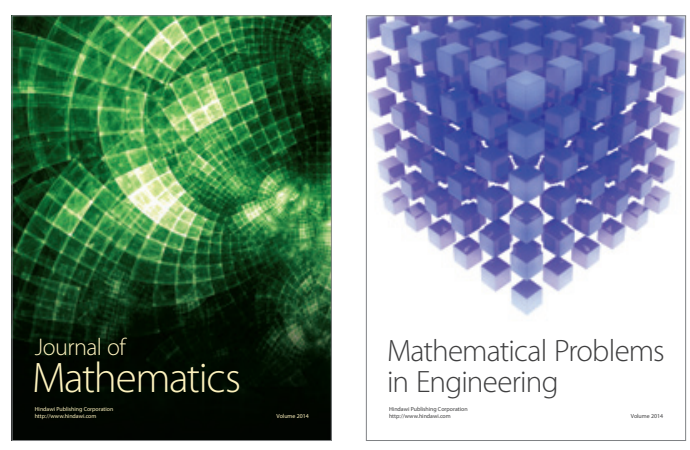

Mathematical Problems in Engineering
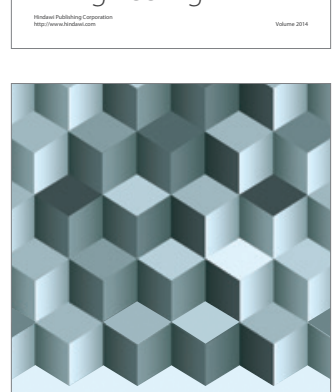

Journal of

Function Spaces
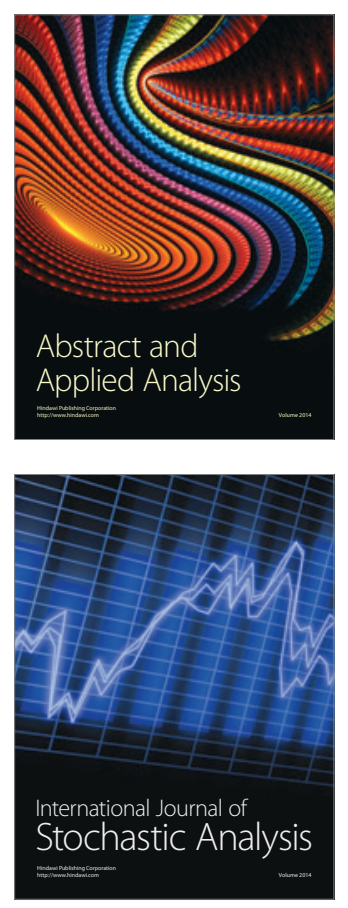

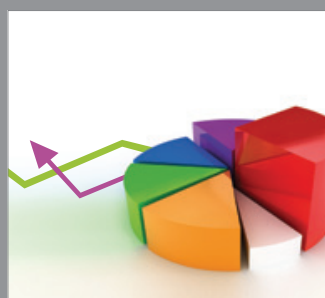

ournal of

Probability and Statistics

Promensencen
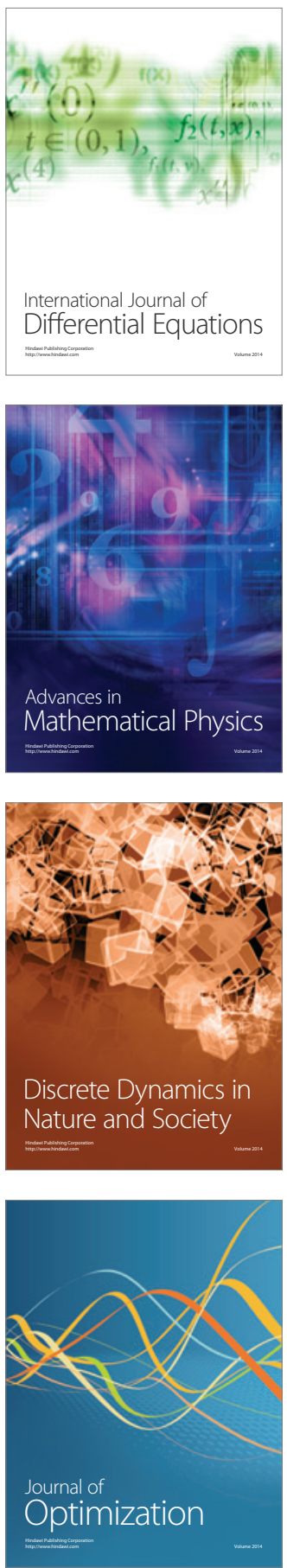